\title{
The Use of Lichenometry for Assessment of the Destruction and Reconstruction of Buddhist Sacred Walls in Langtang Valley, Nepal Himalaya, following the 2015 Gorkha Earthquake \\ Steven H. Emerman (Email: StevenE@ uvu.edu), Department of Earth Science, Utah Valley University, Orem, Utah 84058, USA
}

Lightning Summary

Six months following the 2015 Gorkha earthquake in Nepal, hree fully intact mani walls hosted large crustose lichens hat were not previously present. The most likely explanation was that mani walls had already been reconstructed using previously interior blocks as exterior locks. Moreover, the original mani wall was found $170 \mathrm{~m}$ from its previous location. This research raises the possibility that many Himalayan religious structures are not the original structures, but are replicates that are

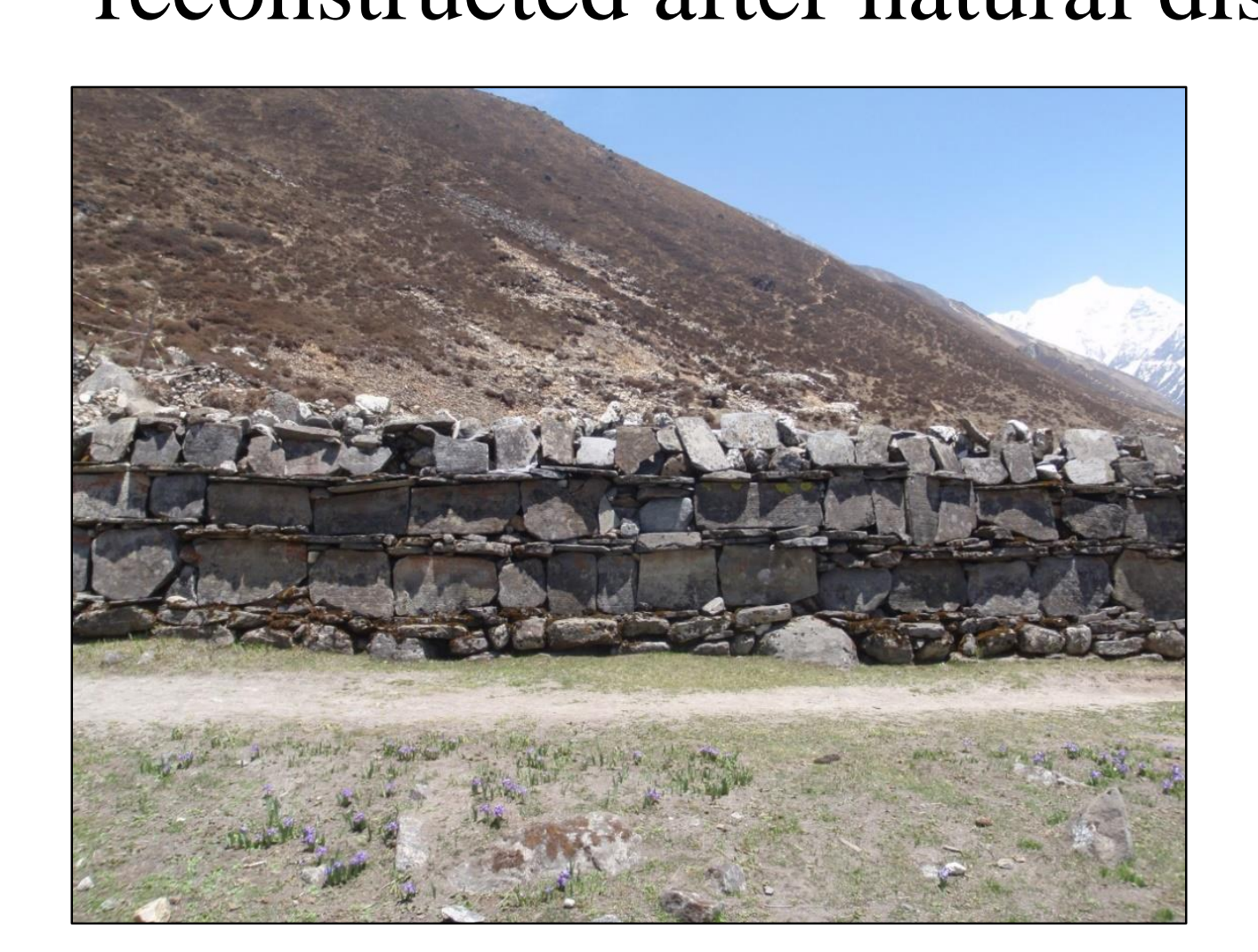

Fig. 1 Buddhist sacred walls, called mani walls, are common in Langtang Valley
(Nepal Himalaya and especially between
the site of the former Langtang Village and Kyanin Gompa. This Lananin wall is
adjecent to the monastery in Ky adjacent to the monastery in Kyanjin
Gompa (Mani Wall No. 80). The mani
wall suffered no apparent damage froo wall suffered no apparent damage f fion
2015 Gorkh a arthquake, although
monastery was heavily damaged.

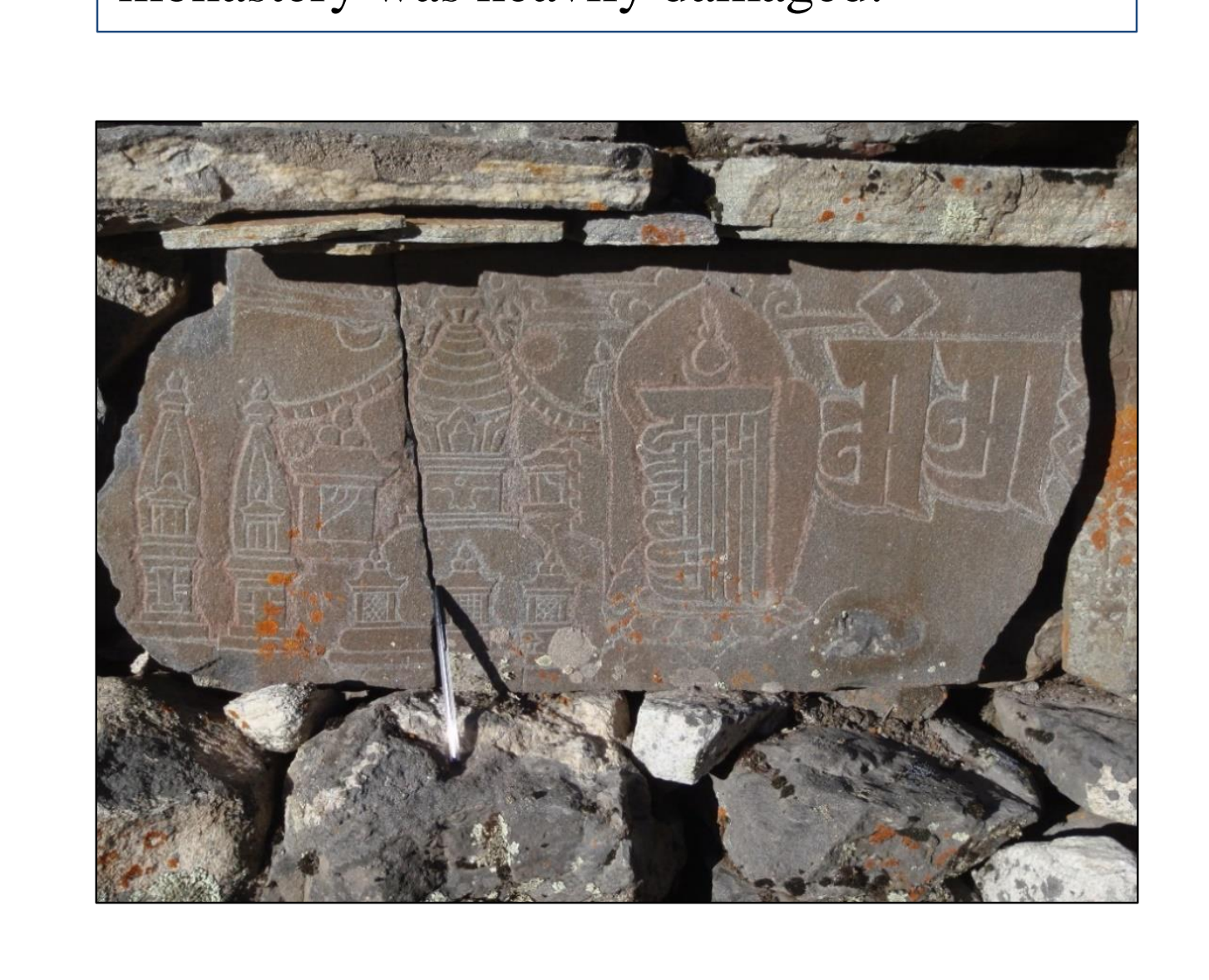

Fig. 3 Many of the mani wall blocks
include carved imagery (Mani Wall No

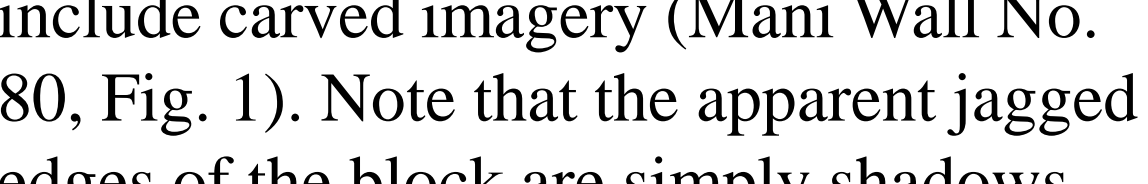

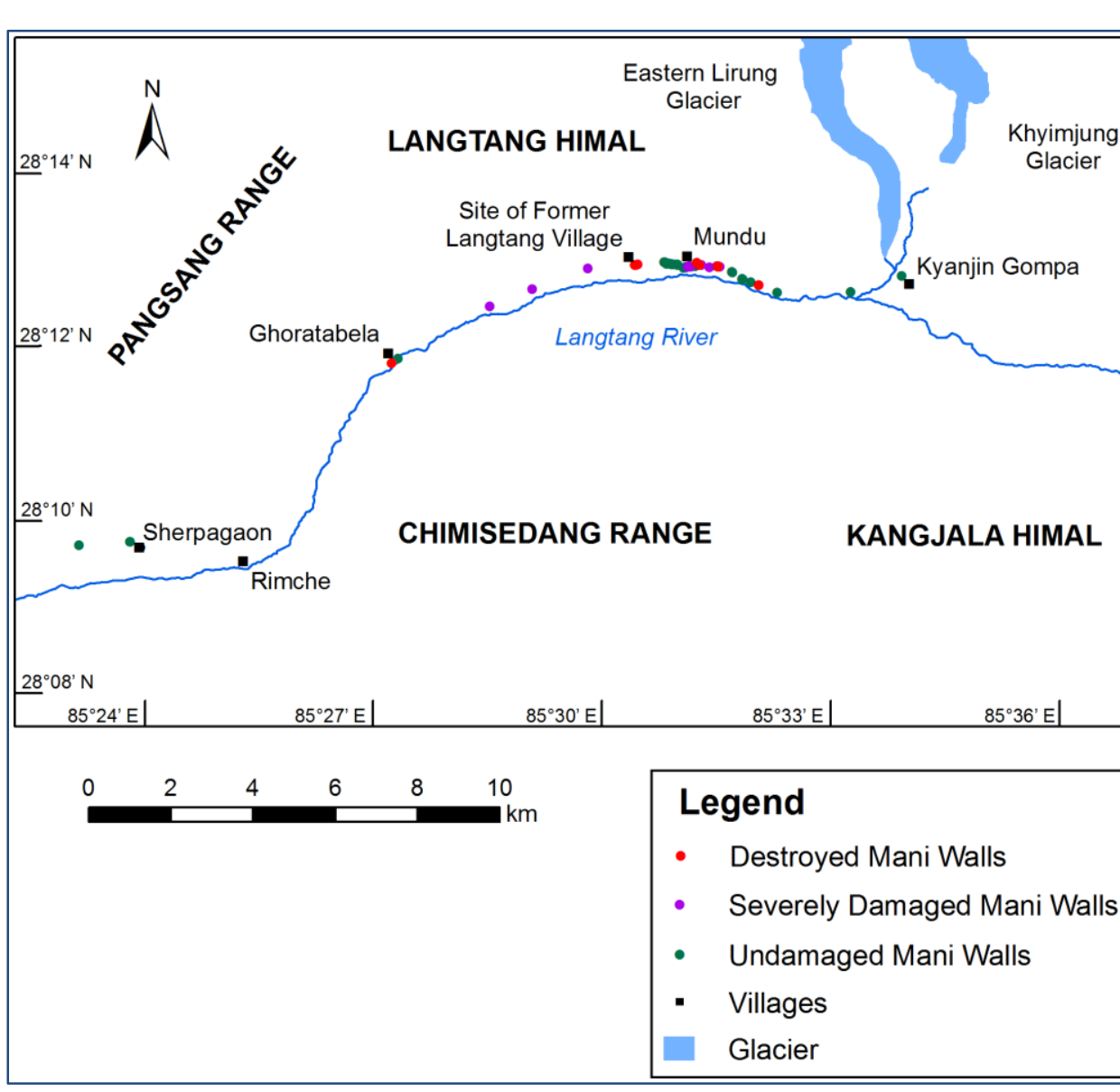

Fig. 5 Out of 80 mapped mani walls in Langtang Valley, $12(15 \%)$ were
completely yestroyed and 16 (2.2\%) were
severely damaged. The remainder suffered severely damaged. The remainder suffered
no opparent damage, athough there was
evidence that at least four had been rebuilt.

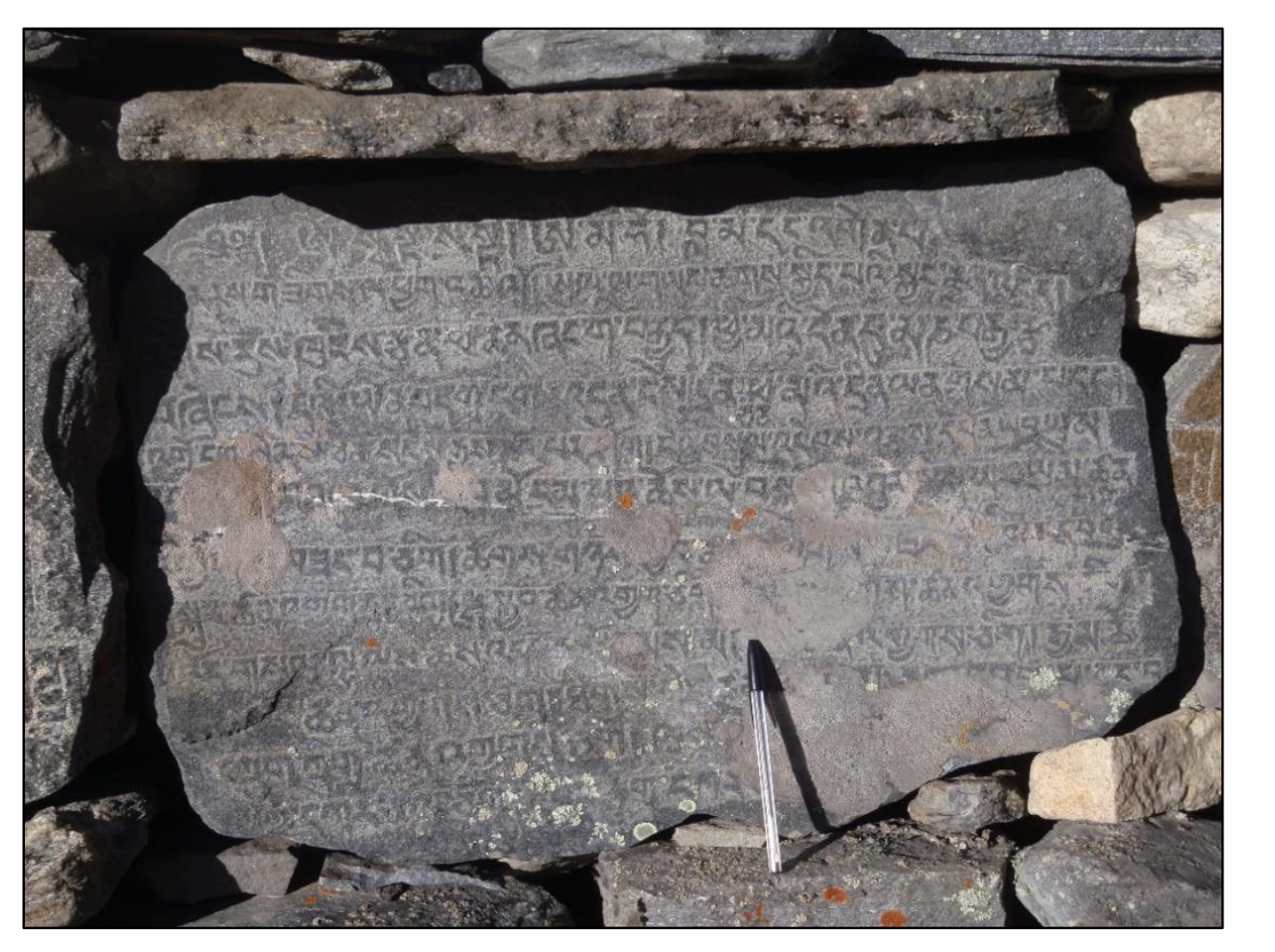

Fig.2 Many of the mani wall llocks
include carved text in Tibetan leteres
Mar

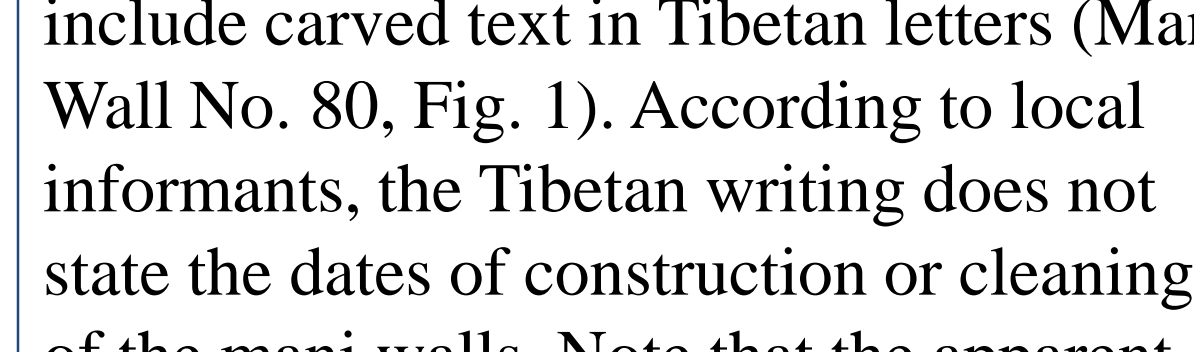

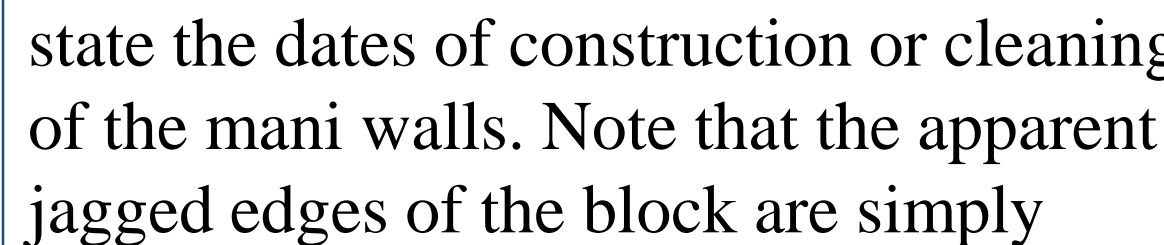

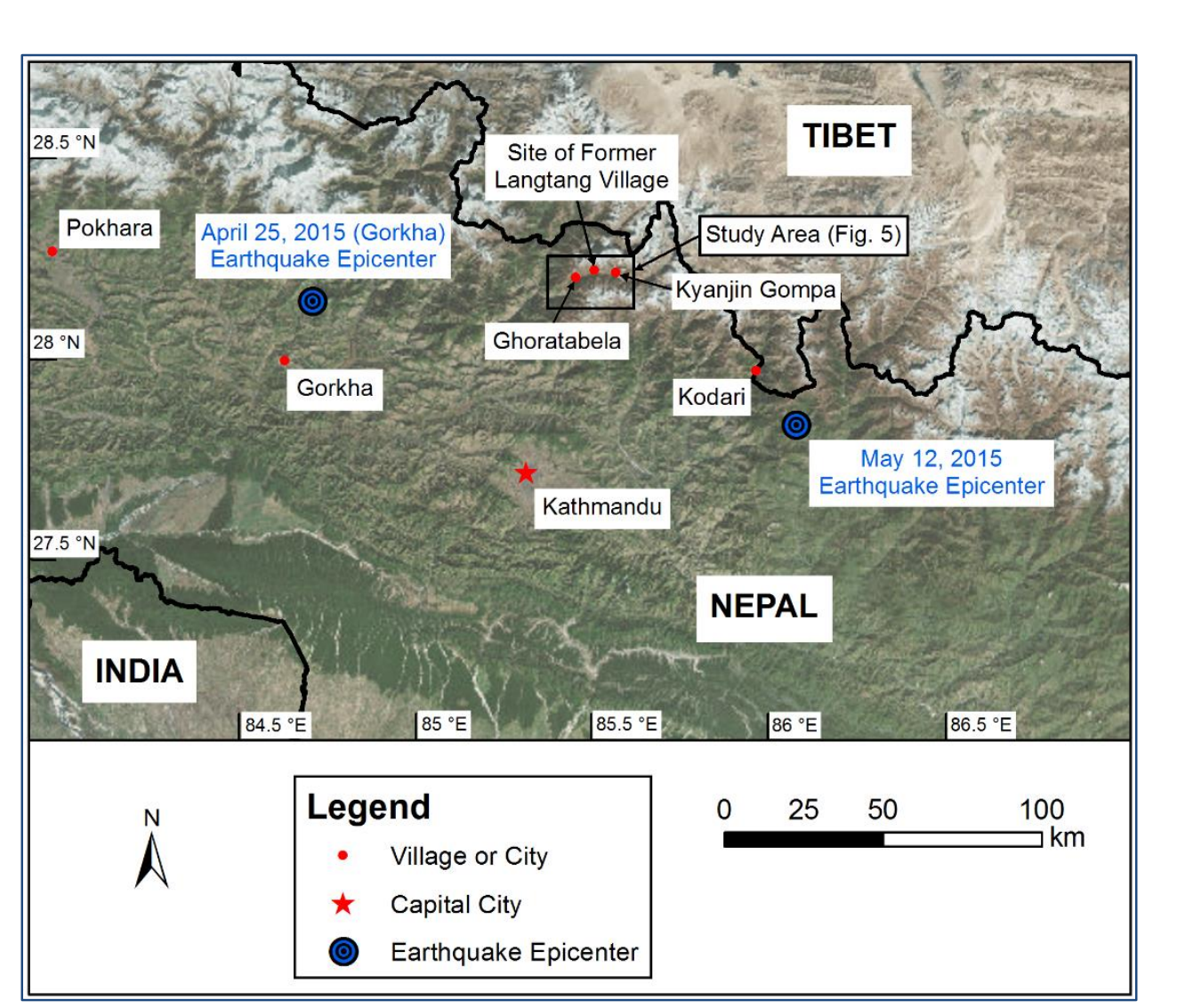

Fig. 4 The 2015 Gorkha earthquake
caused massive landslidini in Langatang
Valley, resulting in loss of life and the Valley, resulting in loss of life and the
destruction of villages and cultural
artifacts.

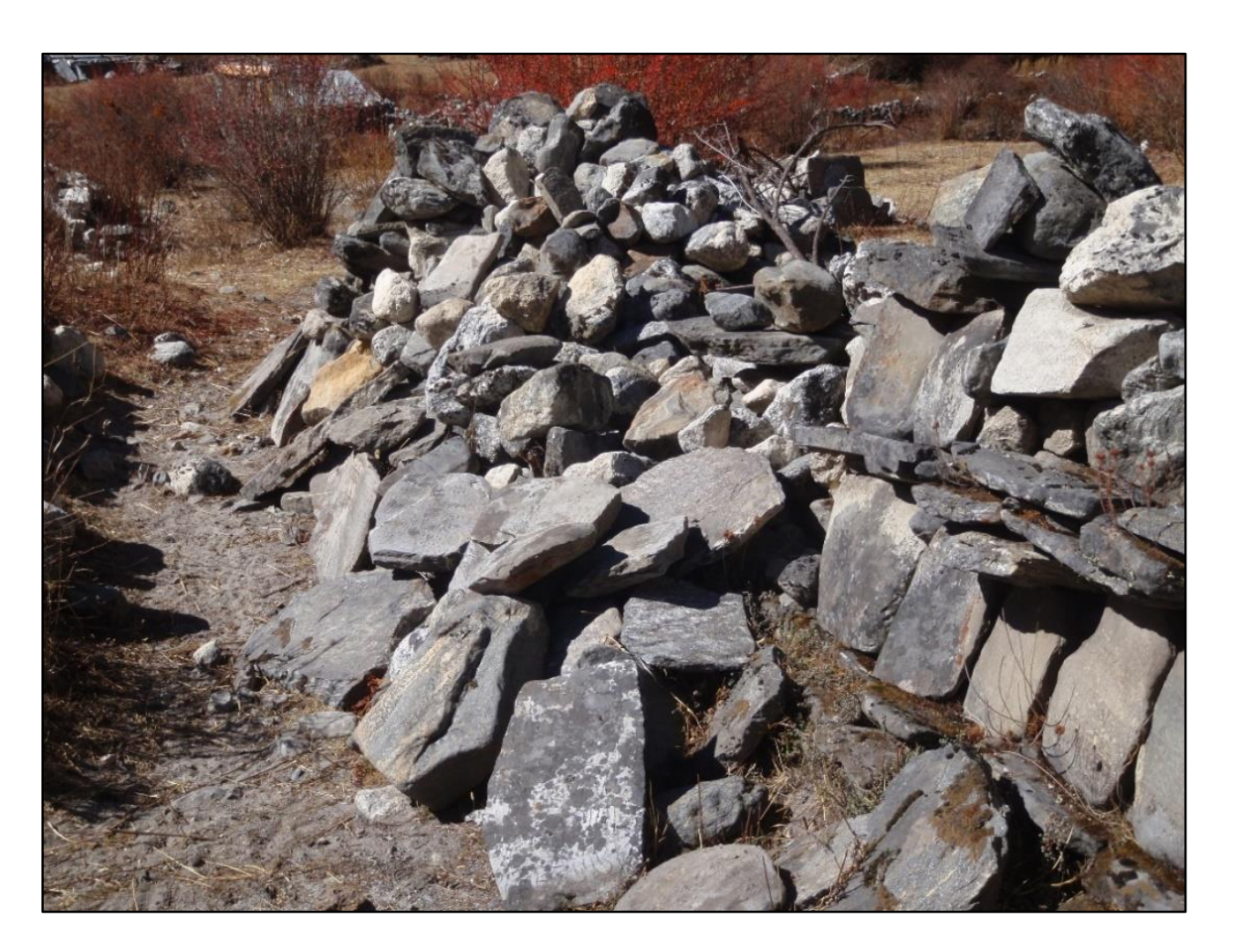

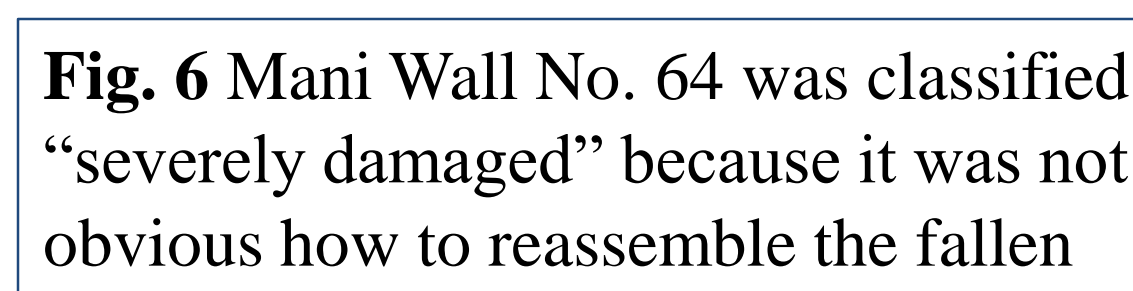

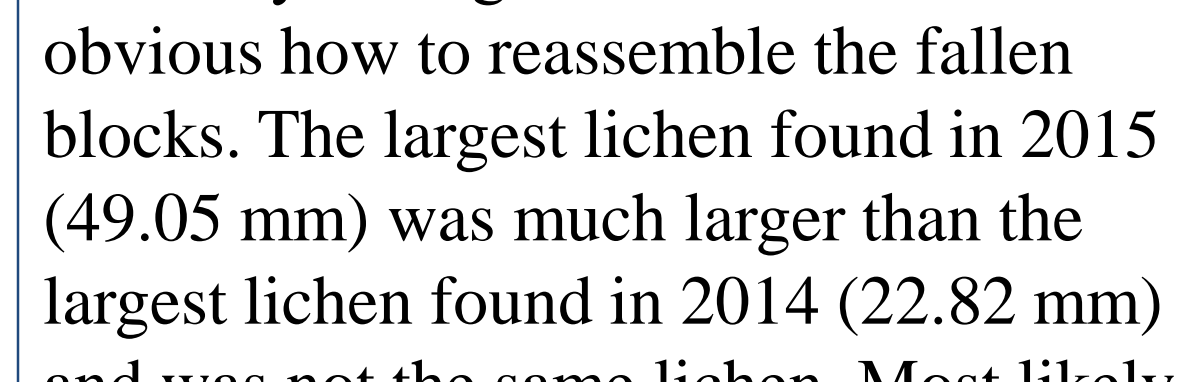

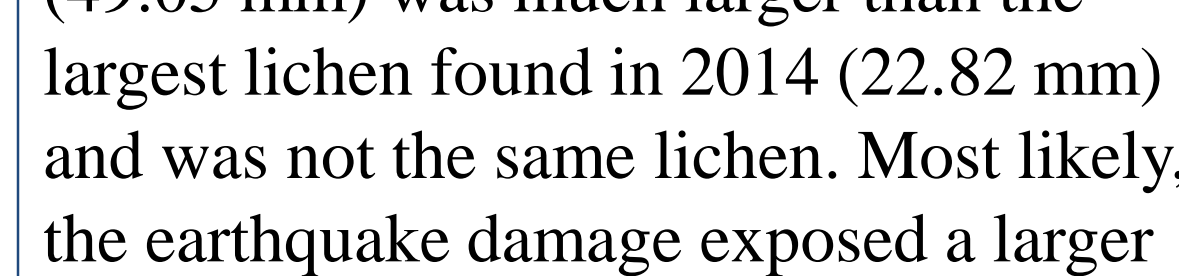

Introduction

Materials and Methods

Mani walls, Buddhist sacred walls constructed of carved blocks with Tibetan letters and elaborate imagery, are common in Langtang Valley, Nepal Himalaya
(Figs. 1-3). Fieldwork in 2009-2014 involved mapping and photographing all 80 mani walls, measuring and photographing all occurrences of the crustose lichen
Rhizocarpon geographicum, and interviewing local informants regarding the history and traditions of the mani walls. The consensus of the informants wa hat the mani walls were constructed 400-600 years ago and that the original nd had never been cleaned of lichen. Based on a locally-developed growth curve, the oldest lichen on a mani wall dated only to 1942. On April 25, 2015, Nepal was struck by an earthquake of moment magnitude 7.8 with epicenter 2 accompanied by massive destruction of the temples, of $\sim 9,000$ was

accompanied by massive destruction of the temples, monuments, and other
religious and cultural heritage of Kathmandu Valley. An icefall-debris avalanch

triggered by the Gorkha earthquake completely buried Langtang Village, killing
t least 350 people (Figs. $4-5$ ). After learning of the tragedy in Langtang Valley, it occurred to the author that the date of the earliest mani wall was, within th strike Neppl prior to the 2015 Gorkh earthquake. The concurrence of dates led to the following questions:

Are Himalayan religious structures actually the original structures or are they replicates that are reconstructed after natural disaster

The objective of this study was to repeat all field work in $L$ angtang $\mathrm{V}$ Valley. including mapping and photographing mani walls, measuring the lichens on mani walls, and interviewing the local residents, in order to assess the destruction and possibe
Gorkha earthquake.
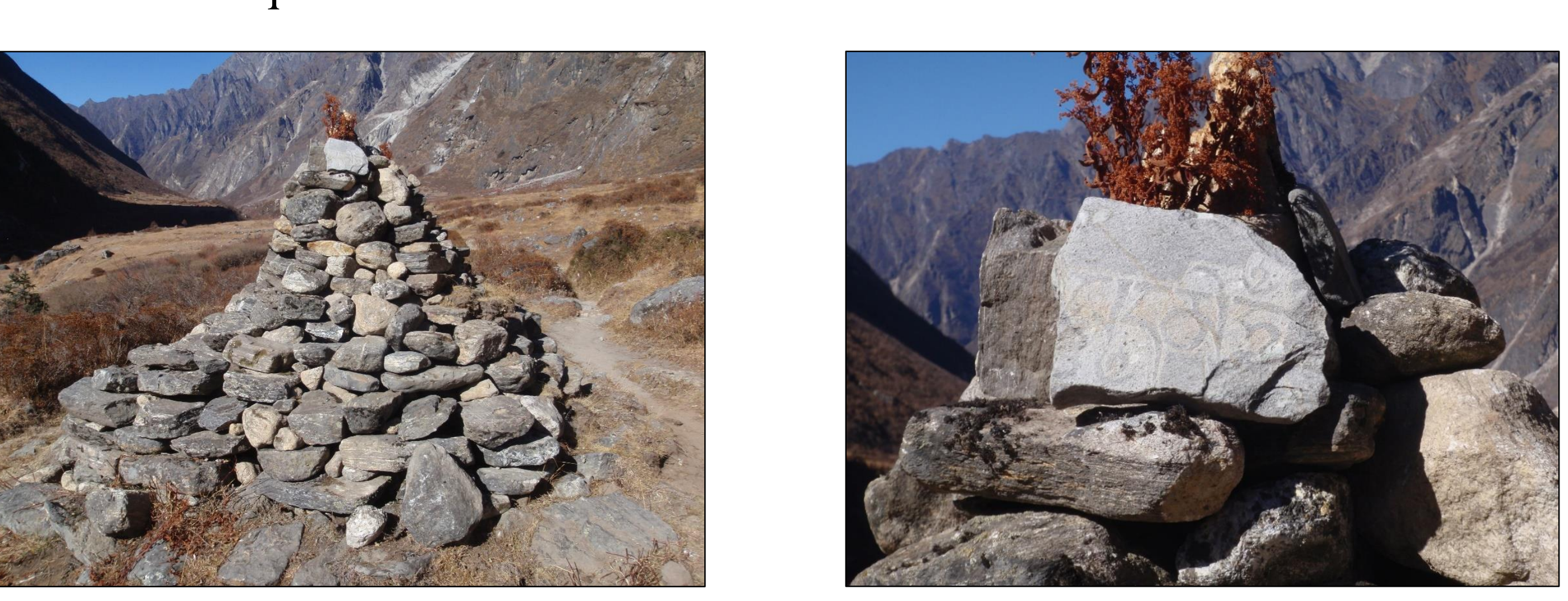

Fig.7 7a Mani Wall No. 77 was mapped in
2009 and 2014, but could not be located in 2009 and 2014 , but could not be located in
2015 . In its placestood a conicis structure
(called a chorten that had been apparently

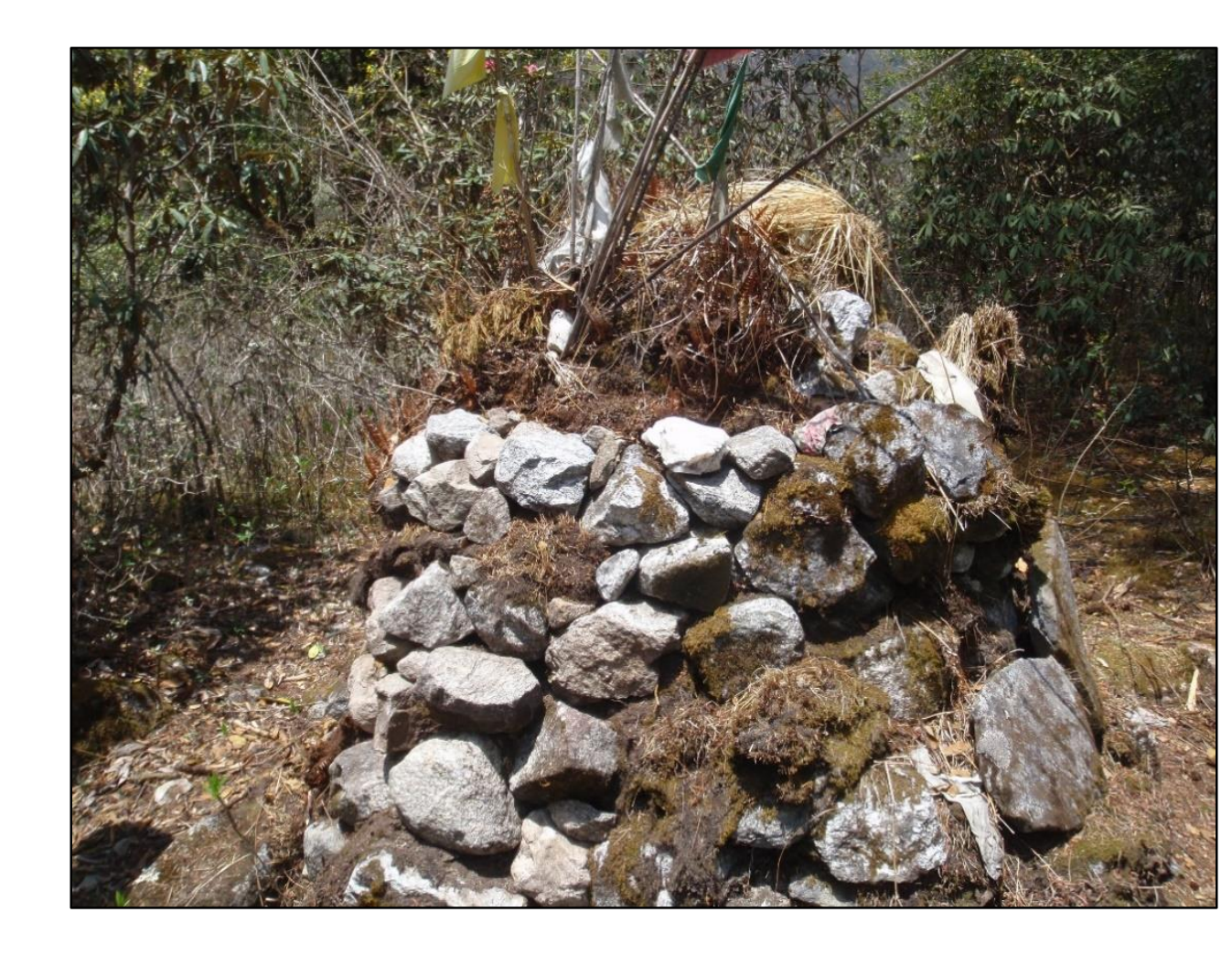

broken block with carved Tibetan letters on the top of the chorten wast probably a
remnant of Mani Wall No. 77 .

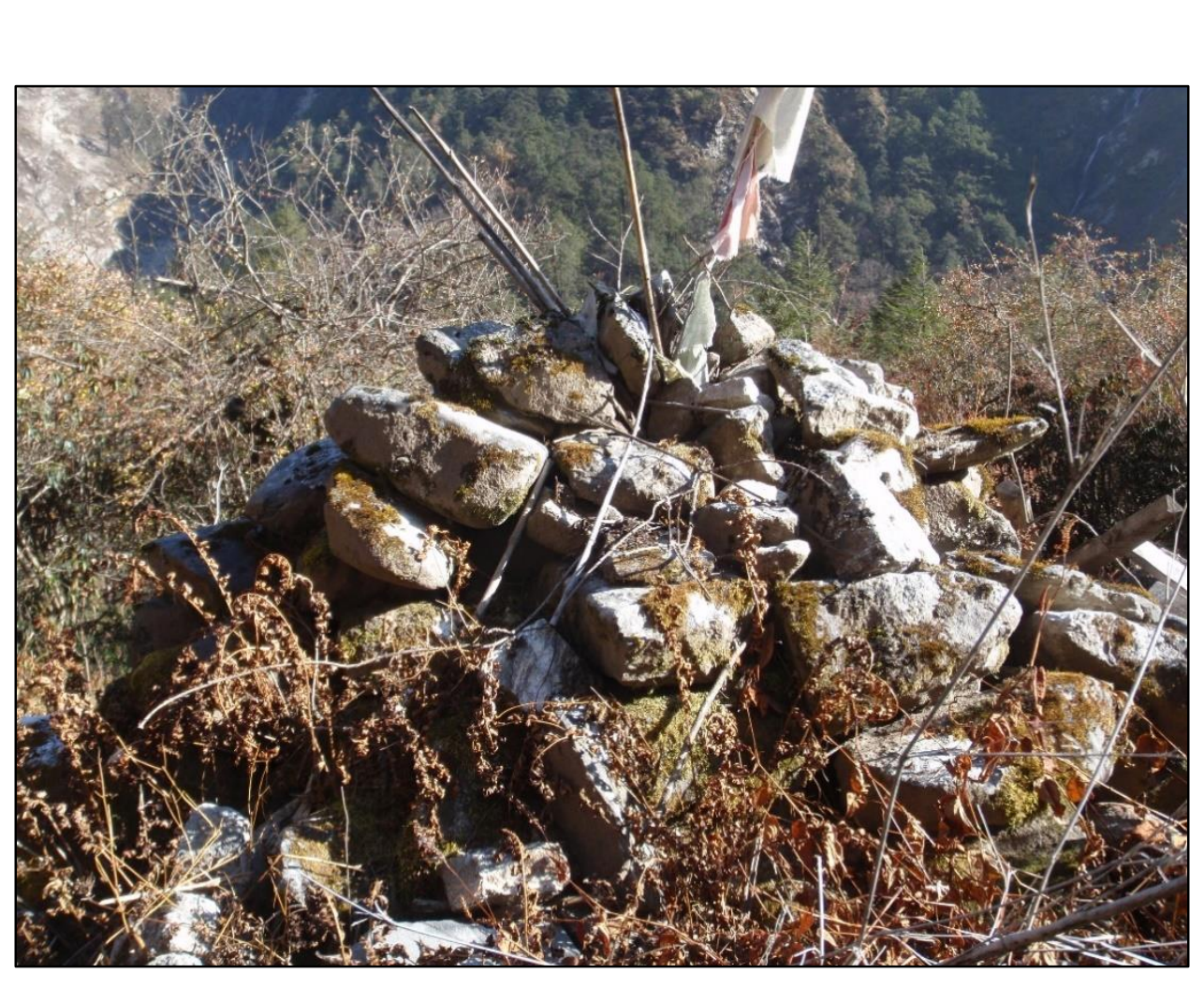
Fig. 8 The appearance of Mani Wall
No. 4 near Ghoratabela, as seen in 2014 ,
was consistent with the oral tradition the was consistent with the oral tradition that
this wasthe original mani wall
(constructed $400-00$ years ago) and had
never been cleaned

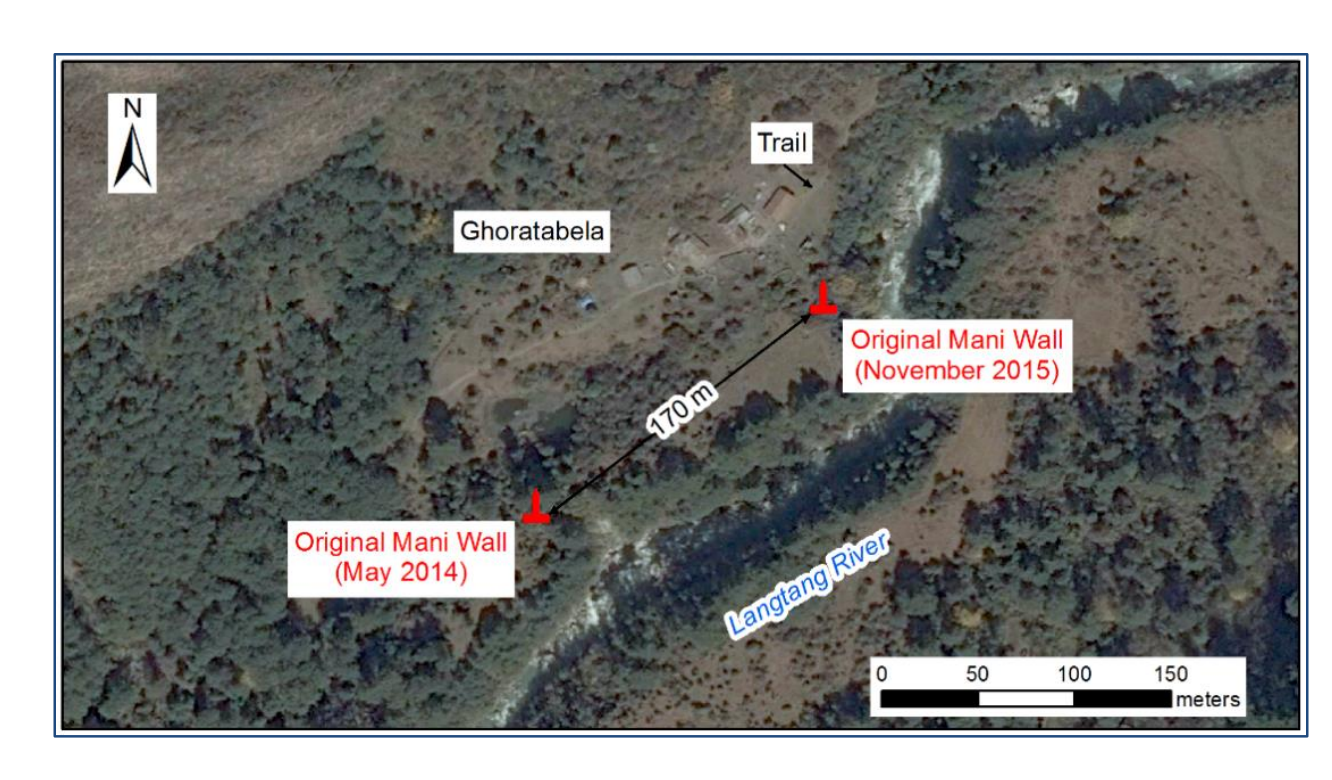

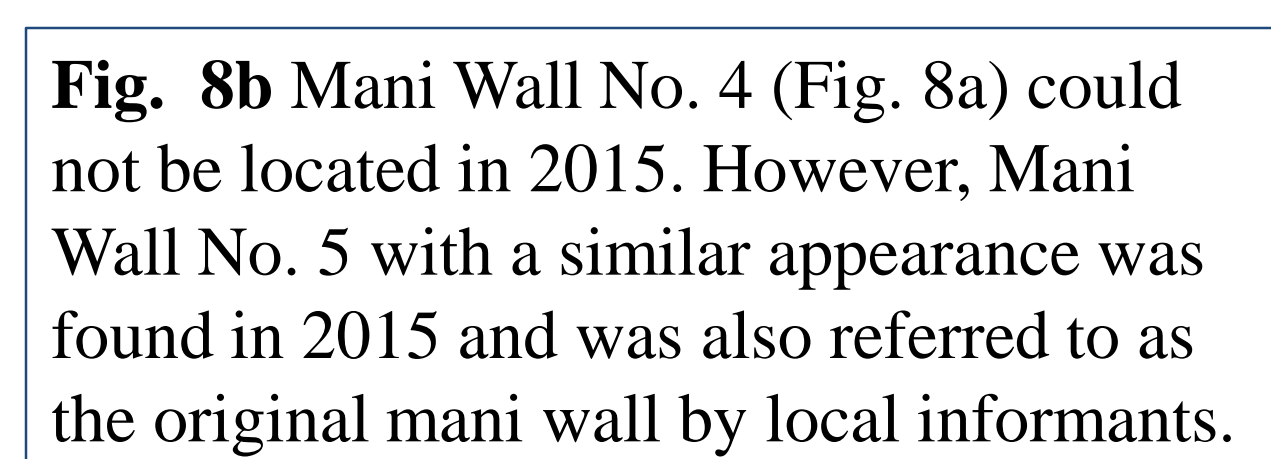

Fig. 9 The original mani wall was

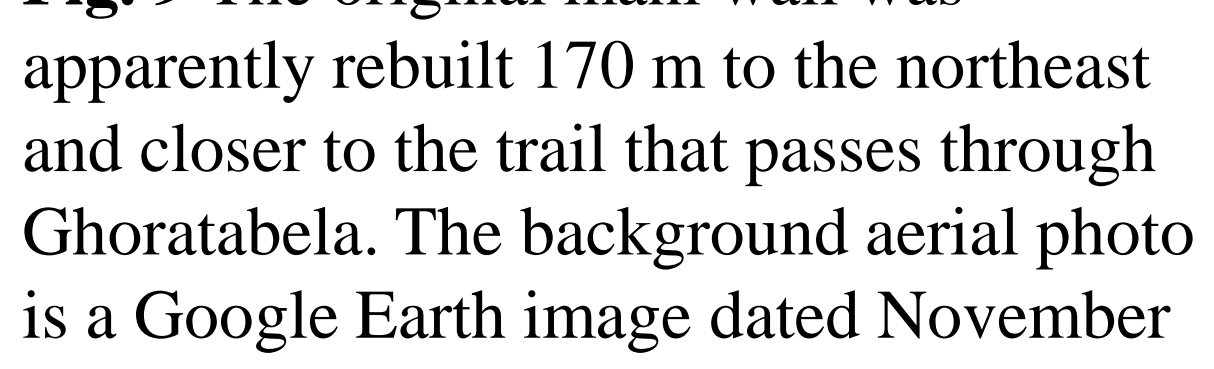
is a Google Earth himage dated Noven
8,2014 (before the 2015 Gorkha 8,2014 (before the 2015 Gorkha
earthuake). In November 2015 all
buildings in Ghoratabela were still hea not be located at all. post-earthquake fieldwork was carried out from November 18-25, 2015, almost exactly six months after the earthquake. Additional interviews with loca residents did not follow a fixed set of questions, but concerned the history and
traditions of the mani walls, the maintenance and cleaning of the mani walls, the damage caused by the 2015 earthquake, and any previous damage to the mani slight (3) severe (4) destroyed. No damage meant that no blocks were apparently out of place. Slight damage meant that one or more blocks had slipped out of place, but it was farly obvious how to reconstruct the mani wall. Severe damage reconstruct the mani wall (Fig. 6). A mani wall was classified as destroyed if it

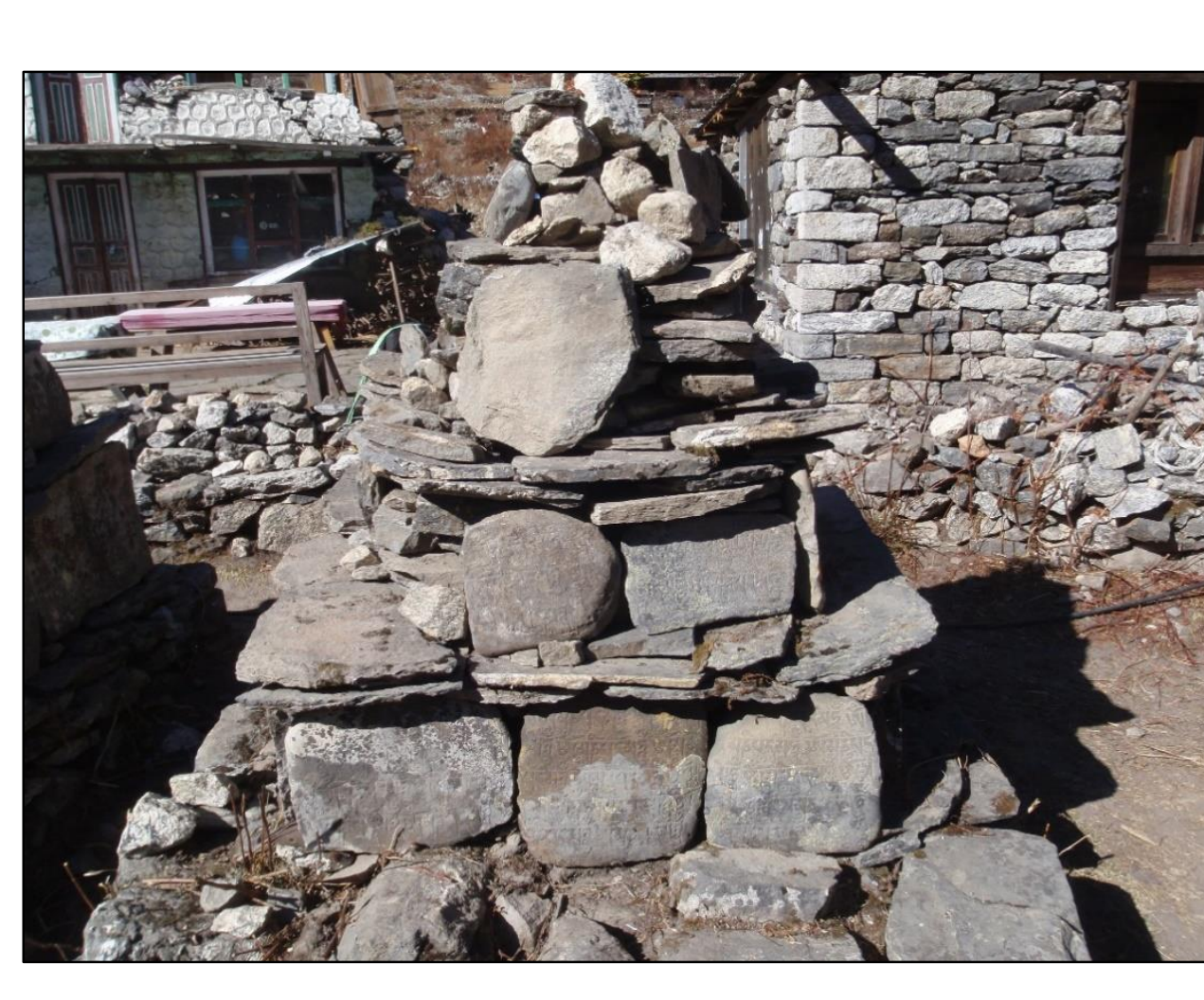

Fig. 10 Atthough Mani Wall No. 53 had no apparent damage, the largest lichen in
2015 was $43.42 \mathrm{~mm}$, although no lichens were present in 2009 o o o 2011 . . The most
likely explanation is that the mani wall had already been reconstructeted in unch a way
that a formerly interior block was placed on the exterior of the mani wall. The mant
wall was in the village of Mundu (Fig. 5 ), which was inhabited at the time of
2015 fieldwork (note house in
background) background.

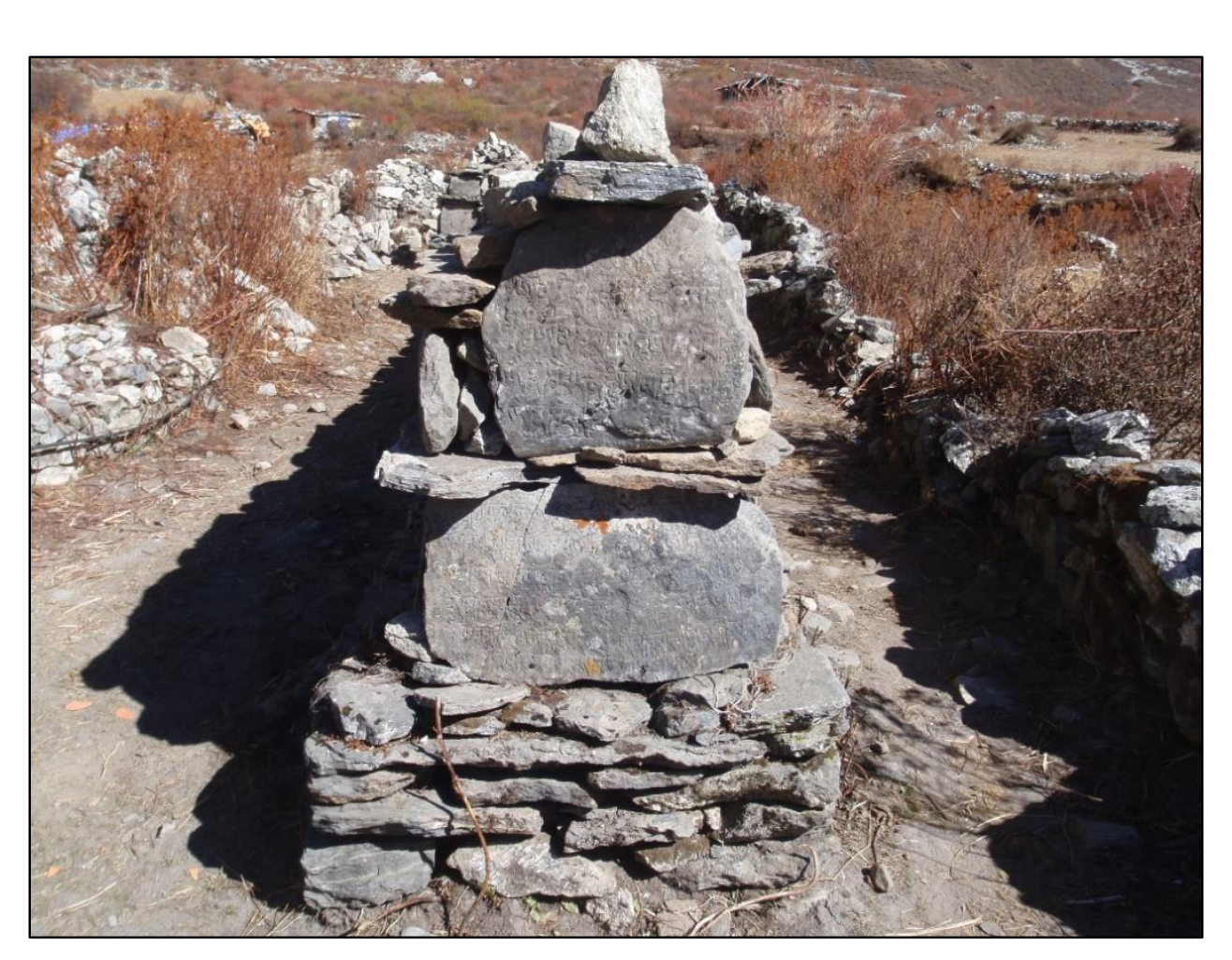

Fig. 11 Although Mani Wall No. 54 had no 2015 was $37.80 \mathrm{~mm}$, although ho holichen,

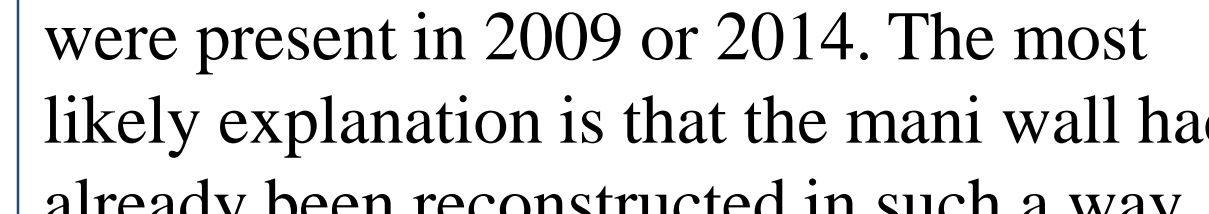
already been reconstructed in such a way
that a formerly interior block was placed that a tormerly interior block was placed
on the exterior of the mani wall The emani
wall wasin the villase of Mundu wall was in the village of Mundu (Fig. 5 ,
which was inhabited at the time of the

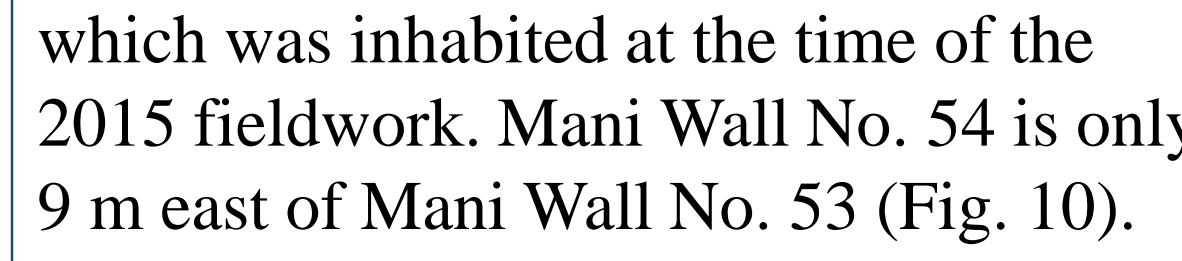

Results

Out of the 80 mani walls in Langtang Valley, it was found that 12 mani walls damaged (Figs. 5-6). At each of three sites of former mani walls (Mani Wal Nos. $67,76-77)$, a chorten was found that was topped by what was apparently a
broken, carved block of a former mani wall (Figs. 7a-b). This combination of a chorten topped by a mani wall block had never been seen by the author befoe anywhere in the Nepal Himalaya and could be regarded as a form of post-
disaster religious art. There were no cases of slightly damaged mani walls and no transition zones between areas of undamaged and either severely damaged or destroyed mani walls (Fig. 5). It is most likely that the mani walls had already
been repaired in cases where it was obvious how to put the blocks back into been repaired in cases where it was obvious how to put the blocks back into
place. The original mani wall in Ghoratabela could not be found among the place. The original mani wall in Ghoratabela could not be found among the
scattered boulders and fallen trees at the location where it had been previously mapped (Mani Wall No. 4, Figs. 5, 8a). However, a similar structure that had no the previous original mani wall and closer to the trail that passes through Ghoratabela (Mani Wall No. 5, Higs. 8b, 9, 10). The easiest mani wall to for several centuries, as opposed to replicating the intricate design of the maintained mani walls (Fig. 1).

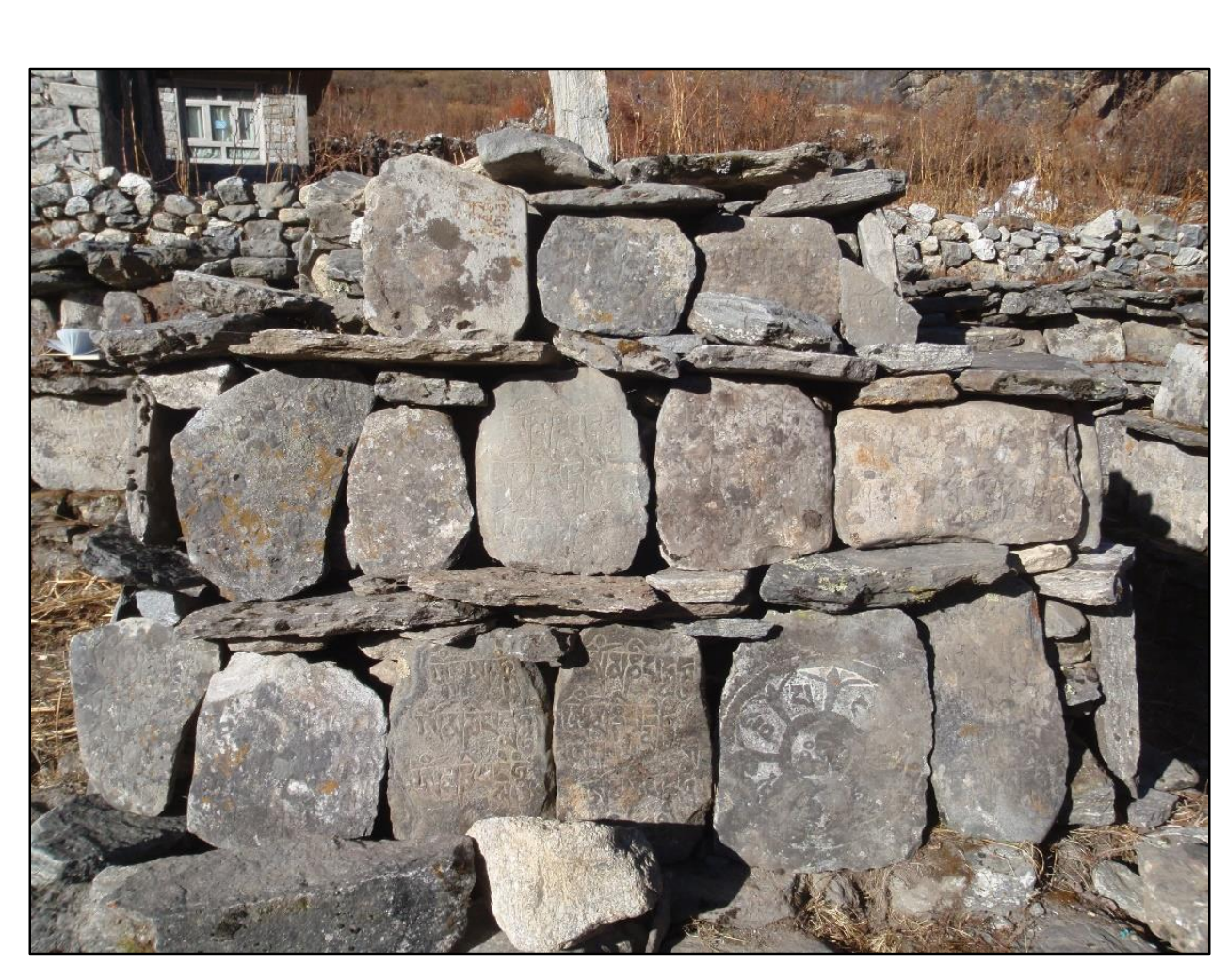

The most challenging observation is the existence of five mani walls on which he largest $R$. geographicum in 2015 was not even present during any previou
fieldwork. Two of the mani walls were severely damaged, but the other three appeared to have suffered no damage at all (Figs. 6, 10-12). Where did these from? The only plausible explanation for the post-earthquake appearance of
previously unseen lichens is that mani wall blocks that were formerly interior heavily bleached with a strong color contrast from white to pale yellow (Fig. 13), as opposed to the bright yellowish-green of healthy $R$. geographicum (Fig.
14). These lichen colors were consistent with a previous history as an interior 14). These lichen colors were consistent with a previous history as an interio block, where only diffuse light with a strong spatial heterogeneity of light
intensity would be present. The three apparently reconstructed mani walls wer

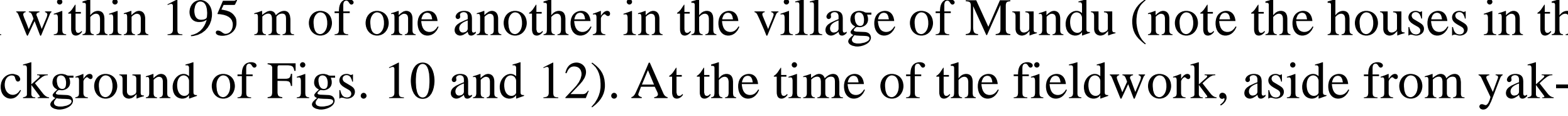
herders living in temporary dwellings above Ghoratabela, Mundu was the only (Fis 5) valitlage in Langtang Valley between Rimche and Kyanjin Gompa ( walls would be reconstructed.
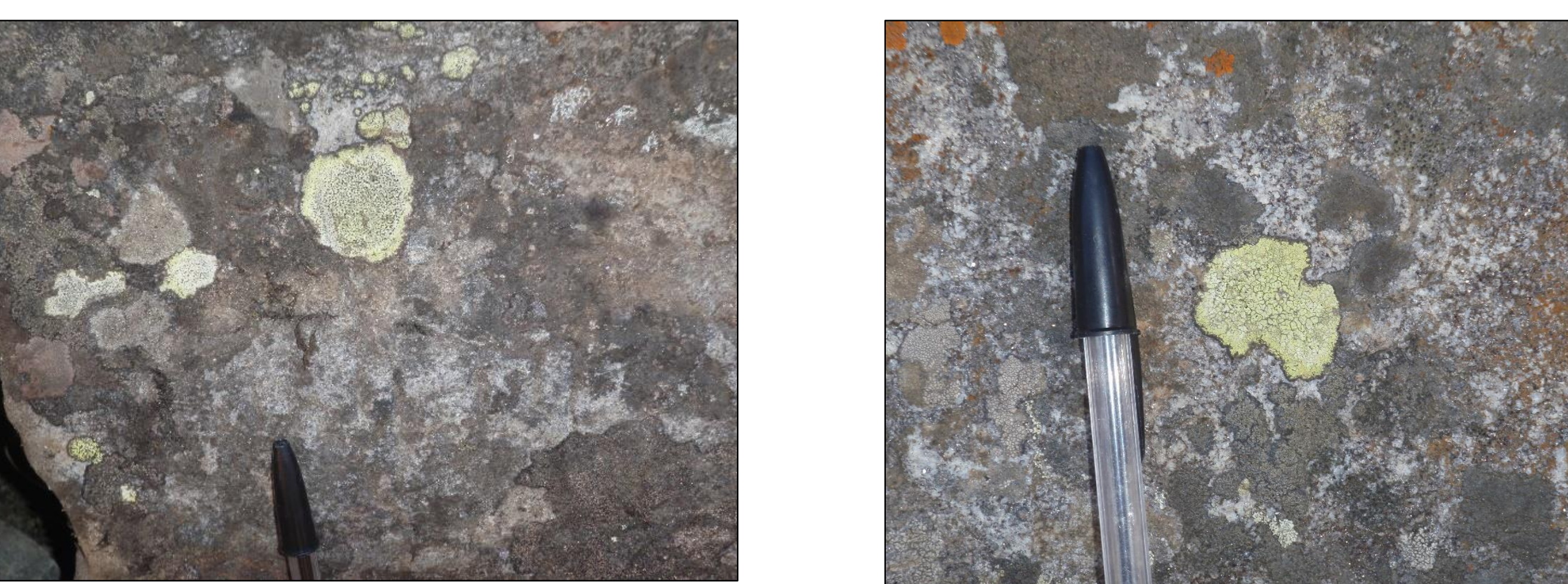

Fig. 13 The lichens found on Mani Wall
No. 54 (Fig. 11) were heavily bleached pale yellow, as opposed to the brigh gellowish-green of healthy Rhizocarpon
geographicum (Fig. 14). The lichen color
Were consistent with a previous history

Fig. 14 Healthy Rhizocarpon

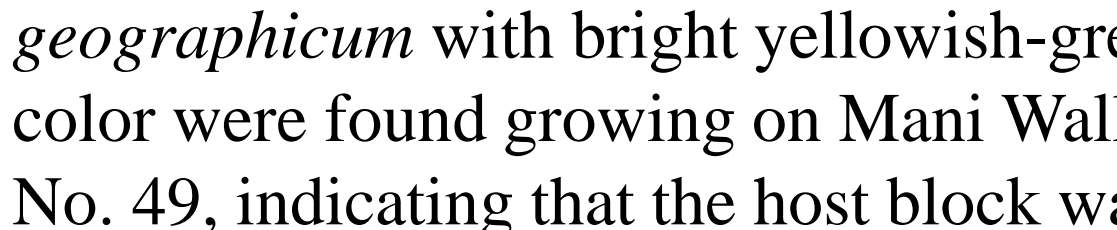
sunlight both before and after the 2015 an interior block, where only diffuse light
with strong spatial heterogeneity of light
Discussion

There are five lines of evidence that, within six months of the 2015 Gorkha o, but not identical to the original design:

The structure that had been called the original mani wall by pre-earthquale informants could no longer be located. After the earthquake, a structure w original mani wall and was referred to as the original mani wall by postoriginal mani wall and
earthquake informants

Three apparently undamaged mani walls in a single inhabited village were
hosting previously unseen $R$. geographicum individuals. The exterior block locks, which (n) mani walls.

Two apparently undamaged mani walls had no R. geographicum, although . previously. This rate of cleaning contrasted with pre-earthquake fieldwork
that showed that lichens were completely removed from mani walls at the rate of three mani walls every five years.
Four mani walls showed a decrease in the size of the largest lichen over the walls at the rate of five mani walls every five years.
he last two observations would not constitute evidence for reconstruction by was consistent with the bleached appearance of the lichens. previous 18 months. This rate of cleaning contrasted with pre-earthquake themselves, but they help to support a pattern of extra attention paid to the man 